Somnologie $2017 \cdot 21: 101-109$ DOI 10.1007/s11818-017-0117-5

Eingegangen: 13. Januar 2017

Angenommen: 21. April 2017

Online publiziert: 15. Mai 2017

(c) Der/die Autor(en) 2017. Dieser Artikel ist eine Open-Access-Publikation.

CrossMark

Heinrich Garn' (DD - Marco Castrucci' - Bernhard Kohn' - Christoph Wiesmeyr' Klaus Dittrich ${ }^{1} \cdot$ Gerhard Kloesch ${ }^{2} \cdot$ Robert Stepansky $^{3} \cdot$ Markus Wimmer $^{4}$. Andrijana Stefanic ${ }^{2} \cdot$ Marion Böck $^{2} \cdot$ Magdalena Mandl $^{4} \cdot$ Osman Ipsiroglu ${ }^{5}$. Dieter Grossegger ${ }^{6} \cdot$ Manuel Kemethofer $^{2} \cdot$ Stefan Seidel $^{2}$

${ }^{1}$ AIT Austrian Institute of Technology GmbH, Wien, Österreich

${ }^{2}$ Medizinische Universität Wien, Wien, Österreich

${ }^{3}$ Krankenhaus der Barmherzigen Brüder, Wien, Österreich

${ }^{4}$ Kepler Universitätsklinikum GmbH, Linz, Österreich

${ }^{5}$ University of British Columbia, Vancouver, Kanada

${ }^{6}$ Dr. Grossegger \& Drbal GmbH, Wien, Österreich

\title{
Detektion periodischer Beinbewegungen im Schlaf
}

\section{Ein Vergleich von Elektromyographie und 3D-Videoanalyse}

\begin{abstract}
Periodische Beinbewegungen können fragmentierten und nicht erholsamen Schlaf bewirken, in der Folge zu häufigen Krankenständen und neuropsychologischen Defiziten führen und sollten daher möglichst frühzeitig diagnostiziert und behandelt werden. Da die routinemäßig verwendete Elektromyographie eine Reihe von Problemen aufweist, haben wir untersucht, wie sich eine berührungslose 3D Analyse von dem konventionellen EMG von linkem und rechtem $M$. tibialis anterior unterscheidet. Dieser Beitrag präsentiert Ergebnisse einer Studie an 27 PLM-Patienten aus der klinischen Routine.
\end{abstract}

\section{Hintergrund und Fragestellung}

\section{Medizinisches Problem}

Periodische Beinbewegungen im Schlaf („periodic leg movements in sleep“, PLMS), früher auch als „nächtlicher Myoklonus" bezeichnet, sind durch wiederholte Beinbewegungen in kurzem Zeitabstand charakterisiert [14]. Führen diese Bewegungen zu Schlaflosigkeit (Insomnie) oder erhöhtem Schlafdruck (Hypersomnie), die nicht durch andere Ursachen erklärt werden kann, so liegt eine „periodic limb movement disorder“ (PLMD) vor. Die PLMD ist auch durch einen PLMS-Index >15/Stunde definiert. Fragmentierter und nicht erholsamer Schlaf kann in der Folge zu häufigen Krankenständen und neuropsychologischen Defiziten führen und sollte daher möglichst frühzeitig diagnostiziert und behandelt werden.

Bewegungsstörungen treten auch bei anderen schlafbezogenen Erkrankungen auf, vor allem beim „Syndrom der ruhelosen Beine“ („restless legs syndrome“, RLS), bei schlafbezogenen Atmungsstörungen und bei der REM-Schlaf-Verhaltensstörung („rapid eye movement sleep behavior disorder", RBD). Bis zu $80 \%$ der Patienten mit RLS haben auch PLMS. Die Art der Bewegungen, ihre Abfolge und ihr Auftreten in der Nacht zeigen vielfach charakteristische Merkmale der jeweiligen Erkrankung auf. [7].

Die Prävalenz von PLMS in der Allgemeinbevölkerung beträgt etwa 4-11\% [7]. Sie steigt ab dem mittleren Erwachsenenalter an und erreicht $25-58 \%$ in der älteren Bevölkerung [7]. Unterschiede zwischen den beiden Geschlechtern sind nicht bekannt. Verschiedene Faktoren begünstigen das Auftreten von PLMS, wie das bereits genannte RLS, die Narkolepsie, obstruktive Schlafapnoen, RBD,
Neuropathien sowie die Einnahme oder der Entzug diverser Psychopharmaka [7].

\section{Beschreibung periodischer Beinbewegungen}

Periodische Beinbewegungen sind kurze, stereotype und wiederholte Bewegungen. Sie werden oft als rhythmisches Strecken der großen Zehe und Dorsalflexion des Fußgelenks beschrieben, mit oder ohne Beugung von Knie oder Hüfte. Die wichtigsten Merkmale der PLM sind ihre „Periodizität" und ihr wiederkehrendes Auftreten. „Periodizität“ ist dabei nicht im streng mathematischen Sinn zu verstehen. Der Schwankungsbereich der Intervalle zwischen den einzelnen Bewegungen (,inter-movement interval“, IMI) ist groß, wobei sich die meisten Bewegungen etwa alle 20-30s wiederholen. Manchmal gehen sie mit kortikalen Arousals oder sogar einem kurzen Aufwachen einher. Man nimmt an, dass die PLMs die Ursache von fragmentiertem Schlaf, nicht erholsamem Schlaf und Tagesschläfrigkeit sind [3, 12].

Die Intensität der PLMs variiert von subtilen zu großräumigen, raschen Kontraktionen beider Beine, die auch zu einer Ganzkörperbewegung führen können. Eine Serie von PLMs beginnt oft mit schwachen Bewegungen, die dann 


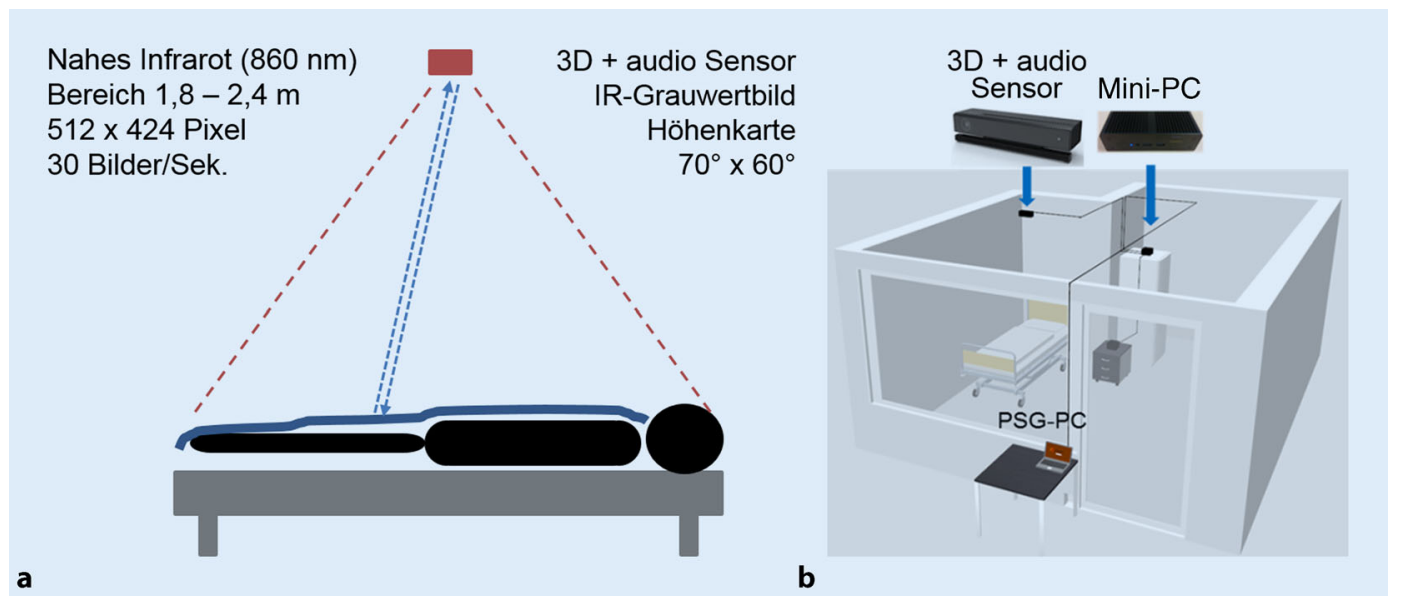

Abb. 1 Anordnung im Schlaflabor. a Sensor über dem Bett; $\boldsymbol{b}$ typischer Aufbau im Schlafraum graduell an Intensität zunehmen, bis ein Arousal oder eine Ganzkörperbewegung folgt. An diesem Punkt kann die Serie enden, von vorne beginnen, oder eine andere Form annehmen.

Obwohl die Bewegungen überwiegend in den Beinen auftreten, können auch die oberen Extremitäten z. B. wiederholte Beugungen des Ellenbogens ausführen. Eine oder beide Seiten des Körpers können beteiligt sein, und die Bewegungen können von einer Seite zur anderen wechseln. Die meisten PLMPatienten zeigen aber beidseitige Bewegungen.

PLMs im Non-REM(„non-rapid eye movement")-Schlaf nehmen in der Intensität $\mathrm{ab}$ und in der Dauer zu, wenn der Schlaf tiefer wird. Im REM-Schlaf hingegen sind PLMs weniger intensiv, weniger häufig oder gar nicht vorhanden. Die Bewegungen treten auch in Serien auf, die sich minutenlang wiederholen können.

\section{Merkmale periodischer Beinbewegungen in der Elektromyographie}

In Schlaflaboratorien werden Beinbewegungen mittels Oberflächen-Elektromyographie (EMG) Elektroden an linkem und rechtem M. tibialis anterior detektiert.

Nach den Kriterien der World Association of Sleep Medicine (WASM) [18] und American Academy of Sleep Medicine (AASM) [2] sind Beinbewegungen nur dann periodisch, wenn sie als Teil einer Serie von zumindest vier aufeinanderfolgenden Einzelbewegungen („leg movements“, LM) auftreten, mit einer Dauervon 0,5 bis $10 \mathrm{~s}$, einem IMI von 5 bis $90 \mathrm{~s}$, und einer Amplitude, die das vor der Bewegung anliegende EMG-Signal um mehr als 8 Mikrovolt übersteigt. Für die Diagnosestellung werden verschiedene Maßzahlen definiert:

- PLM-Index (PLMI) [12, 17]: Anzahl der PLM-Ereignisse pro Stunde Schlaf

- PLMS pro Stunde Schlaf [18]: „Number of leg movement events occurring during sleep that meet PLM criteria divided by the number of hours of sleep with leg movement recording (PLMS/h).“

Bei Kindern gilt ein PLM-Indexvon mehr als 5 als pathologisch, bei Erwachsenen mehr als 15.

Für alle PLM-Detektionen mittels EMG gilt, dass sie nur das Vorhandensein einer Bewegung anzeigen können, jedoch keinerlei Auskunft über Art und Ausmaß der Bewegung geben. Hier kann die Video-Polysomnographie (Video-PSG) Abhilfe schaffen, wobei eine visuelle Inspektion der Schlafvideos jedoch extrem personal- und zeitintensiv ist und daher in der klinischen Routine nur in sehr begrenztem Umfang durchgeführt werden kann.

\section{\) EMG und PSG registrieren die Anzahl, nicht aber die Art und das Ausmaß einer Bewegung}

PLMS zeigen sowohl bei Kindern als auch bei Erwachsenen starke Variationen von
Nacht zu Nacht [6]. Diesem Problem kann man durch ein Heimmonitoring begegnen und mehrere Nächte hintereinander aufzeichnen. Dafür stehen einerseits mobile Polysomnographiesysteme zur Verfügung, andererseits Aktigraphen/Aktometer. Es gilt aber auch hier, dass immer nur das Auftreten von Muskelaktivitäten registriert wird, nicht aber die Art und das Ausmaß einer Bewegung.

Periodische Beinbewegungen müssen von einigen anderen Phänomenen mit teilweise ähnlichen Erscheinungsformen unterschieden werden: In der Einschlafphase treten oft kurze Muskelzuckungen („hypnic jerks“) auf. Sie sind jedoch nicht repetitiv und nicht periodisch. Nächtliche epileptische Anfälle bestehen häufig aus stereotypen Bewegungen, die aber als Einzelereignisse auftreten und keine Periodizität aufweisen.

\section{Probleme und Einschränkungen der Elektromyographie}

Um alle Körperbewegungen vollständig erfassen und diese nach Möglichkeit auch voneinander unterscheiden $\mathrm{zu}$ können, müsste man eine wesentlich größere Anzahl an EMG-Elektroden an verschiedene Muskelgruppen kleben. Während bei den WASM-Kriterien [18] nur „leg movements“ (Beinbewegungen) betrachtet werden, sprechen die DGN [17] und AASM [2] in den Scoring Rules von „limb movements“ (also Arme und Beine). Für die Erfassung von Beinbewegungen definieren die Scoring Rules der AASM den linken und rechten M. tibialis anterior, für die Arme den linken und 
Somnologie 2017·21:101-109 DOI 10.1007/s11818-017-0117-5

(c) Der/die Autor(en) 2017. Dieser Artikel ist eine Open-Access-Publikation.

\section{H. Garn · M. Castrucci • B. Kohn · C. Wiesmeyr · K. Dittrich · G. Kloesch · R. Stepansky · M. Wimmer · A. Stefanic · M. Böck · M. Mandl · O. Ipsiroglu · D. Grossegger $\cdot$ M. Kemethofer $\cdot$ S. Seidel \\ Detektion periodischer Beinbewegungen im Schlaf. Ein Vergleich von Elektromyographie und 3D- Videoanalyse}

\section{Zusammenfassung}

Hintergrund. Periodische Beinbewegungen können fragmentierten und nicht erholsamen Schlaf bewirken, in der Folge zu häufigen Krankenständen und neuropsychologischen Defiziten führen und sollten daher möglichst frühzeitig diagnostiziert und behandelt werden.

Fragestellung. Welche Vorteile liefert eine berührungslose 3D-Analyse zur Bewegungsdetektion gegenüber der klassischen Elektromyographie (EMG) von linkem und rechtem M. tibialis anterior?

Material und Methoden. Untersucht wurden Schlafaufzeichnungen von 27 Patienten aus drei neurologischen Schlaflaboren. Zusätzlich zur routinemäßigen Video-Polysomnographie (PSG) wurde ein 3D-Sensor über dem Bett verwendet, der das Höhenprofil des Körpers scannt und aus Änderungen Bewegungen detektiert.

Ergebnisse. In den Schlafaufzeichnungen wurden 5710 Beinbewegungen (leg movements, LM) durch 3D-Analyse detektiert und durch Schlafmedizinern verifiziert. Das EMG erfasste davon nur 3317 Ereignisse (58\%). Die restlichen 2393 Beinbewegungen wurden vom EMG nicht detektiert (42\%). Es traten auch 1545 EMG-Aktivitäten auf, ohne dass dabei 3D- oder Videodetektionen beobachtet wurden (32\% der insgesamt 4862 Ausschläge im EMG). Diese können durch technische Artefakte, subtile tonische Muskelaktivität ohne Bewegung oder durch schwache, unter der Decke verborgene Bewegungen verursacht worden sein.

Diskussion. Die 3D-Analyse detektiert auch Beinbewegungen, die von anderen Muskeln als den M. tibialis anterior ausgelöst werden. Artefakte von schlechten Elektrodenkontakten spielen dabei keine Rolle. Insgesamt ergab die 3D-Analyse von Beinbewegungen eine realistischere LM-Anzahl als die EMGbasierte Zählung. Die 3D-Analyse hat daher auch therapeutische Konsequenzen. Ein großer Vorteil gegenüber dem EMG ist die berührungslose Bewegungsdetektion: Sie vereinfacht die Aufnahmeprozedur, verringert die Sensoren am Körper und ermöglicht so ungestörten Schlaf.

Schlüsselwörter

Elektromyographie - 3D-Videoanalyse . Periodische Beinbewegungen im Schlaf . Polysomnographie · Artefakte

\section{Detection of periodic leg movements in sleep. A comparison of electromyography and 3D video analysis}

\begin{abstract}
Background. Periodic leg movements can cause fragmented and non-restorative sleep, lead to frequent sickness absences and neuropsychological deficits and should therefore be diagnosed and treated as early as possible.

Objectives. To determine possible advantages of a contactless 3D analysis for motion detection in comparison to conventional electromyography (EMG) of the left and right tibialis anterior muscle.

Materials and methods. We investigated sleep recordings of 27 patients from three neurological sleep laboratories. In addition to the routine video-polysomnography (PSG), a $3 \mathrm{D}$ sensor above the bed was used, which
\end{abstract}

scanned the height profile of the body and detected movements.

Results. In the 3D video analysis, 5710 leg movements (LM) were detected and verified by somnologists. The EMG captured only 3317 events (58\%). The remaining 2393 leg movements were not detected by the EMG (42\%). In addition, 1545 EMG-activities without $3 \mathrm{D}$ or video detection occurred (32\% of 4862 deflections in the EMG). These could have been caused by technical artifacts, subtle tonic muscle activity without motion, or weak movements that were hidden under the blanket.

Discussion. 3D analysis also detects leg movements caused by muscles other than the tibialis anterior muscle. Artifacts from poor electrode contacts do not play any role. Overall, 3D analysis of leg movements provided a more realistic LM number than EMG-based counting. Therefore, 3D analysis also has therapeutic consequences. A major advantage over EMG is the contactless measurement since it simplifies the assessment procedure, lowers the number of body-mounted sensors and thereby enables undisturbed sleep.

\section{Keywords}

Electromyography $\cdot 3 \mathrm{D}$ video analysis $\cdot$ Periodic leg movements in sleep . Polysomnography . Artefacts rechten $\mathrm{M}$. flexor digitorum superficialis oder M. extensor digitorum communis. Für die RBD-Diagnose sieht AASM die Verwendung von Video-PSG-Aufzeichnungen vor, mit denen dann komplexe Bewegungsvorgänge und Vokalisationen im REM-Schlaf festgestellt werden können. Frauscher et al. [8] verwendeten hierfür in einer Studie an 17 RBD-Patienten Elektroden an 13 verschiedenen
Muskeln. Da dies in der klinischen Routine nur sehr eingeschränkt machbar ist, beschränkt man sich in den meisten Schlaflaboren auf die Verwendung von EMG-Elektroden an beiden Mm. tibiales anteriores.

Das EMG kann grundsätzlich nur eingeschränkte Informationen über die tatsächlichen Bewegungsereignisse liefern. Beinbewegungen, die von anderen Mus- keln als z. B. den M. tibiales anteriores verursacht werden, sind im EMG nicht erkennbar. Insbesondere für eine Differenzialdiagnose ist die Ja/Nein-Information, ob eine Bewegung stattgefunden hat oder nicht, unbefriedigend.

Schlechte Elektrodenkontakte machen die EMG-Signale oft unbrauchbar. Dies zeigt sich durch stark erhöhten Rauschpegel oder permanente Span- 


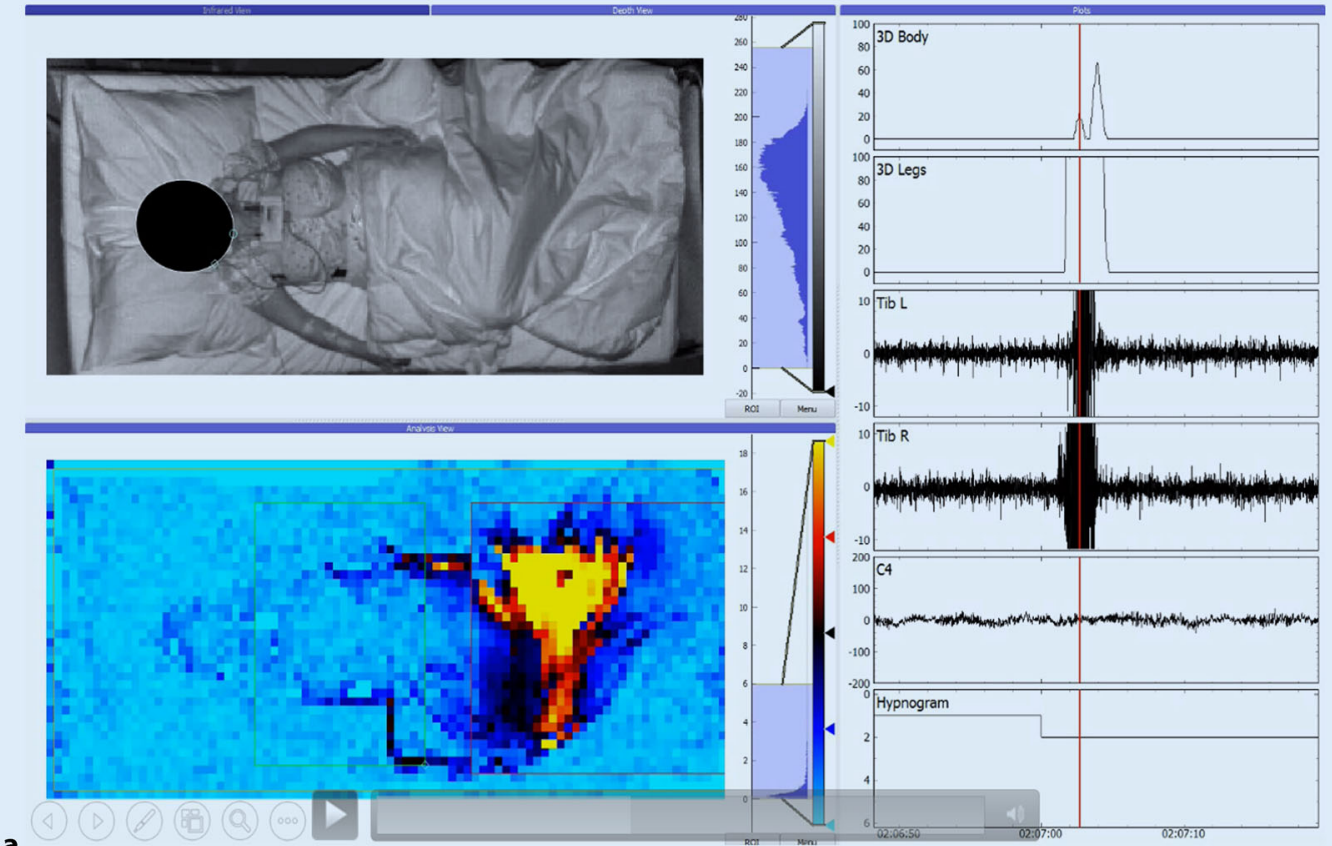

a
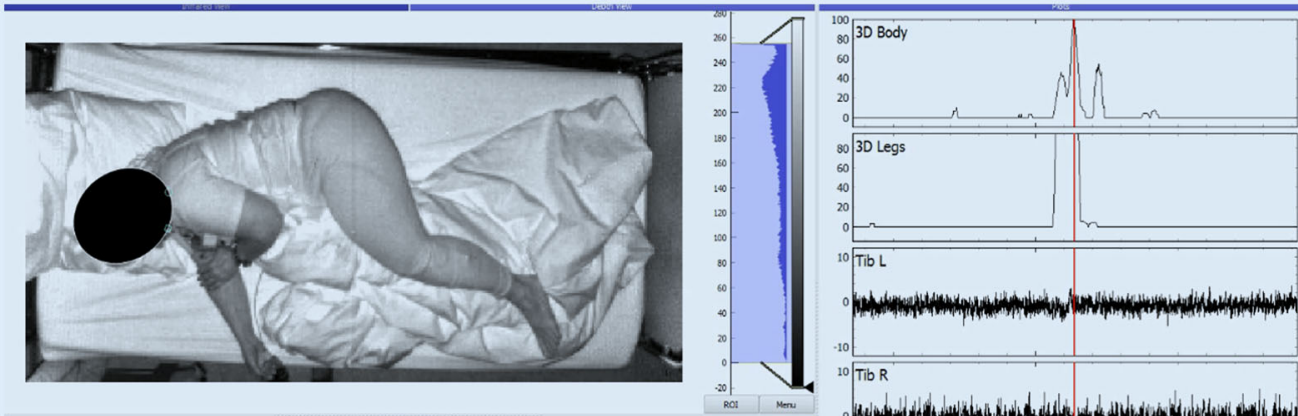

Abb. $2 \varangle$ Zeitsynchroner Vergleich von 3D-Analyse und PSG. Jeweils links: vom 3D Sensor erzeugte Bilder (oben: Nahinfrarotvideo; unten: falschfarbencodierte Bewegungsdetektion [rot = Bewegung]]. Jeweils rechts: Zeitverlauf der 3D und PSG Signale.; 3D Body: detektiert alle Bewegungen die am Körper auftreten; 3D Legs: detektiert Bewegungen der Beine; Tib L, Tib R: EMG-Signale vom linken und rechten $\mathrm{M}$. tibialis anterior; C3, C4: zentrale EEG-Ableitungen; Hypnogram: zeigt das Schlafstadium an (REM = Phase 5). a: 3 D und EMG ergeben übereinstimmende Detektionen; $b$ Detektion in 3D, aber b

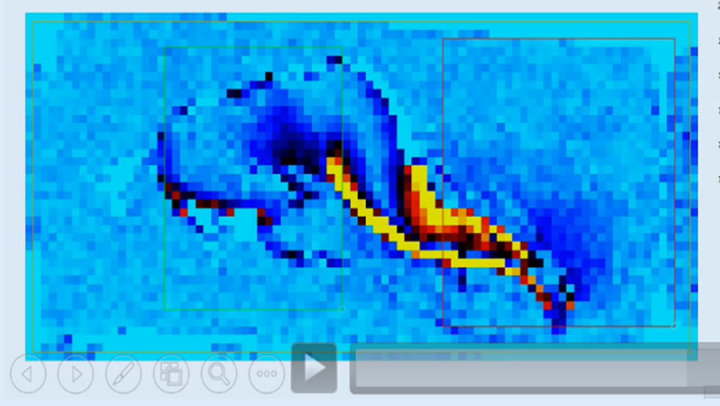

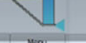
kein Ausschlag im EMG

nungsspitzen. Passiert dies während der Nacht im Schlaflabor, so kann das Personal den Sitz der Elektrode überprüfen bzw. diese wieder neu anbringen, weckt dabei aber den Patienten meist auf. Bei einer Heimanwendung sind die Aufzeichnungen meist verloren.

Generell stellt die Montage und Demontage der Elektroden einen nennenswerten Arbeitsaufwand für das Personal des Schlaflabors dar. Zudem wer- den die Manipulationen von den Patienten zumeist als unangenehm empfunden. Schlaflabors haben begrenzte Kapazitäten, was für die Patienten monatelange Wartezeiten bedeuten kann. Jede Einsparung von Arbeitsschritten könnte daher zur Milderung dieses Problems beitragen. Letztlich stören Elektroden und Kabel die subjektive, wohl aber auch die objektive Schlafqualität. Manche Personen tolerieren dies überhaupt nicht und können diese Untersuchung daher nicht durchführen.

\section{》) Berührungslose Messtechnik könnte die Datenqualität wesentlich verbessern}

Die genannten Probleme sprechen für eine berührungslose Messtechnik. Eine solche könnte eine wesentliche Verbesse- 


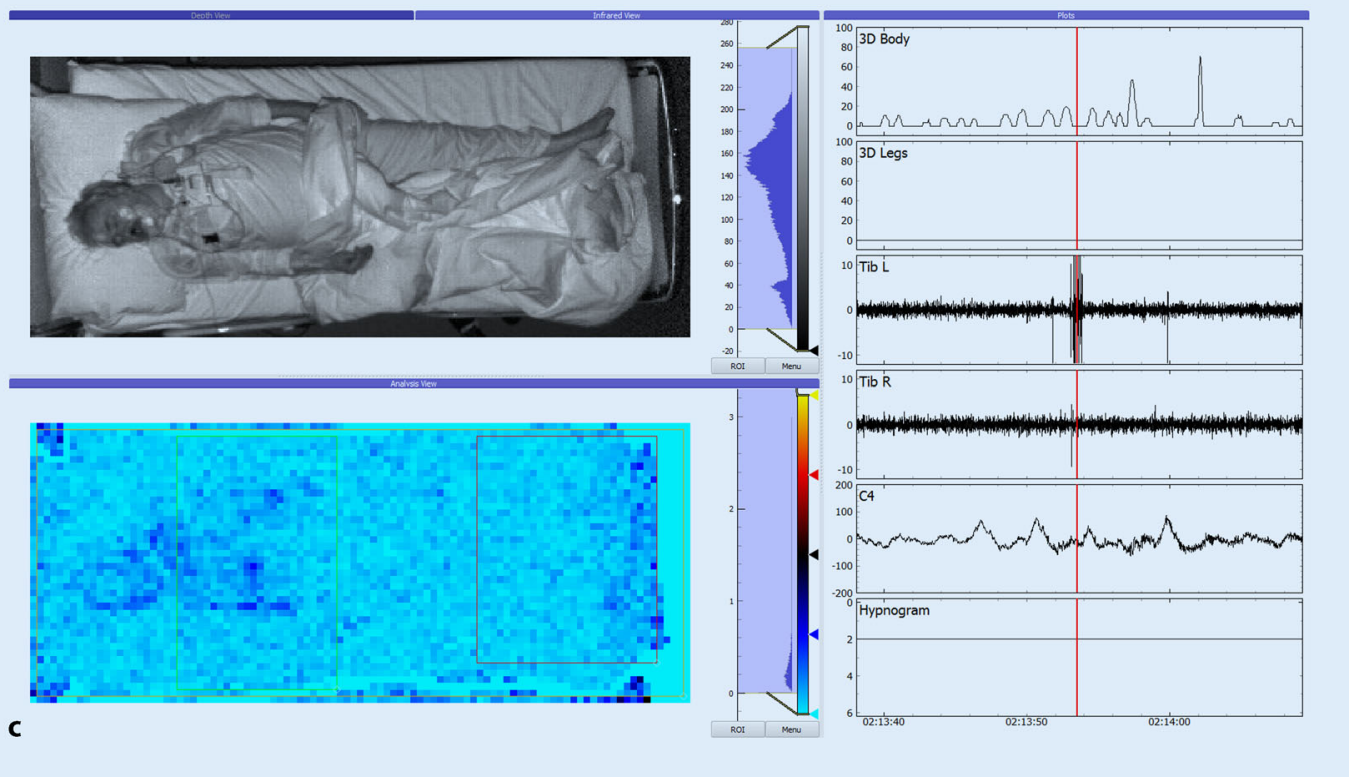

Abb. 2 (Fortsetzung) $\Delta$ Zeitsynchroner Vergleich von 3D-Analyse und PSG. Jeweils links: vom 3D Sensor erzeugte Bilder (oben: Nahinfrarotvideo; unten: falschfarbencodierte Bewegungsdetektion [rot = Bewegung]). Jeweils rechts: Zeitverlauf der 3D und PSG Signale.; 3D Body: detektiert alle Bewegungen die am Körper auftreten; 3D Legs: detektiert Bewegungen der Beine;Tib L,TibR:EMG-Signale vom linken und rechten M.tibialis anterior;C3, C4:zentraleEEG-Ableitungen; Hypnogram:zeigt das Schlafstadium an (REM = Phase 5). c Ausschlag im EMG, aber weder im Video noch in der 3D Detektion sind Bewegungen erkennbar

rung der Datenqualität ermöglichen und die Prozedur vereinfachen. Erwünscht wäre, alle Bewegungen zu erkennen, nicht wie beim EMG nur jene von Muskeln, an denen Elektroden befestigt wurden. Ein kontaktloses Monitoring würde außerdem die Schlafqualität nicht beeinträchtigen. Ein kontaktloses Verfahren könnte auch für das Heimmonitoring wesentliche Vorteile bringen. In der Schlafdiagnose nach heutigem Stand der Technik ist ein solches System jedoch nicht bekannt.

\section{Videobasierte Detektions- methoden}

Bildverarbeitungsverfahren zur Detektion von Körperbewegungen im Schlaf wurden in der wissenschaftlichen Literatur beschrieben, sind aber in der praktischen Anwendung noch nicht etabliert. Verwendet wurden 2D-Grauwertbilder (nahes Infrarot), 3D-Punktwolken, die mit Lichtschnittverfahren oder Stereokameras erzeugt wurden, und 3DTiefenbilder von Time-of-Flight-Kameras.
Bildverarbeitung am $2 D$ nahinfrarotVideo: In praktisch allen Schlaflabors wird heute die Video-PSG verwendet. Es ist daher naheliegend, diese Bilder nicht nur zur visuellen Beobachtung sondern auch zur automatischen Bewegungsdetektion $\mathrm{zu}$ verwenden. Veröffentlicht wurden Methoden zur Schätzung der Pose der Person im Bett, Schätzung der Körperteile, Bewegungsdetektion und Objektverfolgung [1, 3, 11, 15]. Die Detektionsqualität von 2D-Bildverarbeitung auf Infrarotbildern ist aber für die klinische Praxis nicht ausreichend und hat daher keinen Einzug in kommerzielle Produkte gefunden.

Bildverarbeitung von 3D Punktwolken die mit Lichtschnittverfahren erzeugt wurden: Projiziert man ein Lichtmuster auf die Szene und beobachtet dieses mit einer Kamera aus anderem Blickwinkel, so bildet sich die 3D Silhouette darin mit verschobenen Lichtpunkten ab. Treten keine Verdeckungen auf, so kann man aus der Verschiebung die Lage der Punkte im Raum berechnen. Takemura et al. [16] haben z. B. ein System zum Monitoring der Brustkorbbewegungen beim Atmen beschrieben.
Bildverarbeitung auf $3 D$ Tiefenbildern von Time-of-Flight(TOF)-Sensoren: Kohli \& Shotton [10] veröffentlichten 2013 eine Übersicht der verschiedenen Entwicklungen zur Schätzung der Pose eines Menschen mithilfe von Time-of-FlightKameras. Diese Technologie wurde auch eingesetzt, um aufgrund der Atmungsbewegungen des Brustkorbs einer schlafenden Person Apnoen zu detektieren [4, 5]. Eine wissenschaftliche Studie, die mit 3D detektierte Beinbewegungen mit EMGAktivierungen vergleicht, ist bisher nicht bekannt.

\section{Studiendesign und Unter- suchungsmethoden}

\section{Berührungslose 3D-Erfassung der Szene}

Wir verwenden einen TOF-Sensor zur Erfassung der Szene in 3D [13]. Dabei wird schwaches, amplitudenmoduliertes, nicht kohärentes Licht bei einer Wellenlänge im nahen Infrarotbereich $(860 \mathrm{~nm})$ ausgestrahlt. Die Intensität ist so gering, dass sie weit unter den geltenden Sicherheitsgrenzwerten für optische Strahlung 
Tab. 1 Vergleich von visuell verifizierten Detektionen in 3D und Detektionen im EMG

\begin{tabular}{|c|c|c|c|c|}
\hline Bewegungsereignisse & Anzahl & $\begin{array}{l}\text { Mittelwert } \\
\text { Prozent }\end{array}$ & $\begin{array}{l}\text { Standard- } \\
\text { abweichung } \\
(\%)\end{array}$ & $\begin{array}{l}\text { Prozent bei } \\
\text { Einzelpatien- } \\
\text { ten }\end{array}$ \\
\hline $\begin{array}{l}\text { Insgesamt in 3D detektierte Beinbewe- } \\
\text { gungen: }\end{array}$ & 5710 & & & \\
\hline Davon visuell verifiziert & 5710 & 100 & - & 100 \\
\hline Davon Ausschläge im EMG & 3317 & 58 & 19 & 10 bis 85 \\
\hline Davon im EMG nicht detektiert & 2393 & 42 & 19 & 15 bis 90 \\
\hline $\begin{array}{l}\text { Insgesamt Aktivierungen im EMG, die die } \\
\text { AASM bzw. WASM Kriterien erfüllen: }\end{array}$ & 4862 & & & \\
\hline Davon in 3D automatisch detektiert & 3317 & 68 & 20 & 24 bis 100 \\
\hline Davon in 3D nicht detektiert & 1545 & 32 & 20 & 0 bis 76 \\
\hline
\end{tabular}

liegt. Die Oberflächen in der Szene reflektieren die Lichtimpulse zurück, und diese gelangen an eine Matrix von Detektordioden. Mithilfe schneller elektronischer Schaltungen werden dort sowohl die Laufzeit der reflektierten Lichtimpulse (30 Zentimeter entsprechen einer Nanosekunde!) als auch deren Intensität gemessen. Aus der Laufzeit wird der Abstand und aus der Intensität der Grauwert jedes Pixels berechnet. Somit liefert der Sensor sowohl ein 3D-Tiefenbild (Punktwolke) als auch ein 2D Grauwertvideo. Letzteres sieht genauso aus wie von den in Schlaflabors üblichen Nahinfrarot-Videokameras. Die Messungen erfolgen mehrmals pro Sekunde (z. B. 30 Bilder pro Sekunde). Damit können Veränderungen in der Szene sehr gut detektiert werden. Veränderungen der gemessenen Abstände bedeuten Bewegung, die man den einzelnen Körperbereichen zuordnen kann. Somit kann man Bewegungen detektieren. Gegenüber dem EMG ergeben sich allerdings wesentliche Vorteile:

- Bei richtiger Anordnung kann ein

3D-Sensor den gesamten Körper überblicken und somit alle Bewegungen eines Körperteils detektieren, nicht nur Bewegungen, die von den M. tibiales anteriores verursacht wurden. Man erhält somit wesentlich umfassendere Informationen über die Ereignisse im Schlaf.

- 3D bildet den Gesamtkontext des Bewegungsvorgangs ab und ermöglicht damit die softwaretechnische Unterscheidung zwischen Bewegungen einzelner Extremitäten und Ganzkörperbewegungen, z. B. beim Umdrehen. Darüber hinaus können laufend Hinweise für eine Differenzialdiagnose gewonnen werden: Für periodische Beinbewegungen ist z. B. die Zunahme der Intensität der Bewegungen innerhalb einer PLMSerie typisch. Bewegungen eines epileptischen Anfalls hingegen betreffen immer dieselben Körperteile in stereotyper Weise.

- 3D ist nicht von guten Elektrodenkontakten abhängig und liefert daher eine gleichbleibende Datenqualität.

- Ein 3D-Sensor wird einmalig installiert und benötigt keine zusätzlichen Montagearbeiten an Patienten.

\section{Evaluierung}

Beim Einsatz einer neuen Technologie ist es zunächst von Interesse, diese Messtechnik mit herkömmlichen und bereits etablierten Verfahren $\mathrm{zu}$ vergleichen. Hier ist einerseits das EMG zu betrachten, andererseits insbesondere die visuelle Beurteilung von Bewegungsereignissen durch Schlafmediziner in der Video-PSG, d.h. im Nahinfrarotvideo. Relevant sind hierbei die einzelnen Beinbewegungen (LMs), denn nur wenn diese richtig erfasst werden, kann man daraus auch einen korrekten PLM-Index berechnen.

Wir haben in Zusammenarbeit zwischen dem AIT Austrian Institute of Technology, der Universitätsklinik für Neurologie der Medizinischen Universität Wien, der Abteilung Neurologie des Krankenhauses der Barmherzigen Brüder in Wien und der Klinik für Neurologie II des Kepler Universitätsklinikums in Linz eine klinische Studie an PLMS-Patienten durchgeführt [9]. Die Studie wurde von den zuständigen Ethikkommissionen der Medizinischen Universität Wien (Patienten der Medizinischen Universität Wien und am Krankenhaus der Barmherzigen Brüder Wien: EK-Nr. 1091/2014) und des Landes Oberösterreich (Patienten des Kepler Universitätsklinikums: EK-Nr. 254) genehmigt. Die Patienten wurden über die Ziele und den Ablauf der Studie informiert und haben eine Einverständniserklärung unterschrieben. In dem jeweiligen Schlaflabor wurden Sensoren und Computerhardware installiert. - Abb. 1 zeigt die schematische Anordnung des 3D-Video-Aufnahmesystems. Die 3D-Aufnahmen wurden mit den PSG-Aufnahmen zeitsynchronisiert, sodass EMG-Signale und 3D-Videos exakt miteinander verglichen werden konnten.

Aus der laufenden klinischen Routine der drei Kliniken wurden im Zeitraum von August 2014 bis Juli201527 Patienten ausgewählt, die die Diagnose „Periodische Beinbewegungen" hatten und deren 3D- und PSG-Aufzeichnungen vollständig und artefaktfrei vorhanden waren. Es waren 17 Männer im Alter zwischen 20 und 84 Jahren $(52 \pm 32)$ und 10 Frauen im Alter zwischen 17 und 75 Jahren $(46 \pm 29)$. Als 3D-Sensor wurde die Microsoft Kinect One, ein CE-zertifiziertes, kommerzielles Standardprodukt verwendet [13]. Die Kinect hat eine Auflösung von $512 \times 424$ Pixel bei 30 Bildern pro Sekunde. Für die Datenauswertung diente ein von AIT entwickeltes Programmpaket zur Detektion und Lokalisation von Bewegungen in 3D sowie zum Vergleich der Daten aus 3D und PSG.

Um die Sicherheit der Patienten zu gewährleisten, wurden der 3D-Sensor und der daran über USB-3 angeschlossene Mini-PC außerhalb der sogenannten $\mathrm{Pa}$ tientenumgebung angebracht, d. h. in Abständen von mehr als 150 Zentimetern vom Bett. Die Montage des Sensors an der Zimmerdecke erfolgte mit einer zusätzlichen Sicherung gegen Herabfallen.

Die Patienten verwendeten normale, in den Schlaflabors routinemäßig eingesetzte Bettdecken. Diese waren nicht besonders dick, sodass sich Körperbewegungen unter der Decke auch an der Oberfläche dieser Decke abzeichneten. 
Die Videos wurden von erfahrenen Schlafmedizinern visuell begutachtet und manuell annotiert. Alle in 3D automatisch detektierten Beinbewegungen wurden visuell überprüft und in der Visualisierungssoftware einzeln annotiert. Generell ausgeschlossen wurden Ganzkörperbewegungen und Bewegungen im Zusammenhang mit schlafbezogenen Atmungsstörungen. Diese Annotationen dienten als Grundwahrheit für die Evaluierung der 3D-Technologie im Vergleich zum EMG.

Fragestellungen:

1. Wie viele der in der 3D-Analyse automatisch detektierten Beinbewegungen wurden von erfahrenen Schlafmedizinern im Infrarotvideo visuell verifiziert und annotiert?

2. Wie viele der in der 3D-Analyse automatisch detektierten und von erfahrenen Schlafmedizinern via Infrarotvideo verifizierten Beinbewegungen führen auch zu Ausschlägen im EMG?

3. Was bedeuten Beinbewegungen, die in 3D und im Video klar erkennbar sind, vom EMG aber nicht erfasst werden?

4. Gibt es umgekehrt Ausschläge im EMG, bei denen aber weder im Video noch in 3D Beinbewegungen erkennbar sind?

5. Was bedeuten Ausschläge im EMG, bei denen weder in 3D noch im EMG Bewegungen erkennbar sind?
6. Detektiert 3D letztlich mehr oder weniger Ereignisse als EMG?

\section{Ergebnisse}

- Abb. 2 zeigt, wie die Ereignisse in 2D, 3D und EMG gegenübergestellt wurden.

In der Gruppe der 27 Patienten mit PLM wurden in 3D insgesamt 5710 klinisch relevante Einzel-Beinbewegungen (LM) detektiert und visuell verifiziert. - Tab. 1 gibt eine Übersicht der Ereignisse, die mit dem jeweiligen Verfahren detektiert wurden.

Mit 3D wurden praktisch alle klinisch relevanten Beinbewegungen detektiert, vom EMG im Mittel jedoch nur etwas mehr als die Hälfte davon. Dies rührt daher, dass Bewegungen, die von anderen Muskeln als dem M. tibialis anterior ausgelöst wurden, vom EMG nicht erfasst wurden. Dies waren 2393 Beinbewegungen (42\%). Abhängig vom Patienten waren es zwischen 10 und $85 \%$ vollständig erfasster bzw. 15 bis $90 \%$ nicht erfasster Bewegungsereignisse. Insgesamt waren hier die Unterschiede von Patient zu Patient sehr groß, da die Bewegungsmuster individuell eben sehr unterschiedlich sein können.

Es traten auch EMG-Aktivitäten auf, die weder mit 3D noch visuell im Video detektiert wurden: Von den insgesamt 4862 Detektionen im EMG stimmten nur 3317 (68 \%) mit manuellen Annotationen überein. Die restlichen 1545 (32\%) Er- eignisse können nun drei verschiedene Ursachen haben:

a) Technische Artefakte, meist aufgrund schlechter Elektrodenkontakte. Eine konventionelle EMG-Detektionssoftware zählt solche Signale als Beinbewegung, sofern sie die zeitlichen Kriterien erfüllen. Auch bei einer visuellen Kontrolle der Videoaufzeichnung ist meist nicht ersichtlich, ob eine unter der Decke verborgene Bewegung stattgefunden hat oder nicht.

b) Äußerst schwache Bewegungen unter der Decke, die sich weder im Video noch in 3D abzeichnen.

c) Subtile tonische Muskelanspannungen, die zwar EMG-Signale bewirken, aber keine Bewegung auslösen. Solche EMG Signale können auch die AASM- bzw. WASM-Kriterien für „leg movements“ erfüllen.

- Abb. 3 veranschaulicht die Abgrenzung der obigen drei Fälle a/b/c: Bei den 1545 EMG Aktivierungen, bei denen in 3D keine Bewegung registriert wurde, gibt es keine Möglichkeit, die Ursache (a, b oder c) zu eruieren. Damit existiert auch keine Grundwahrheit, auf Basis derer man Sensitivitäten oder Spezifitäten der einen oder anderen Methode angeben könnte.

Fall (a) kann manchmal durch Experten anhand einer visuellen Inspektion des EMG-Signals erkannt werden. In 3D kann der Fall (a) gar nicht passie-

Hier steht eine Anzeige. 


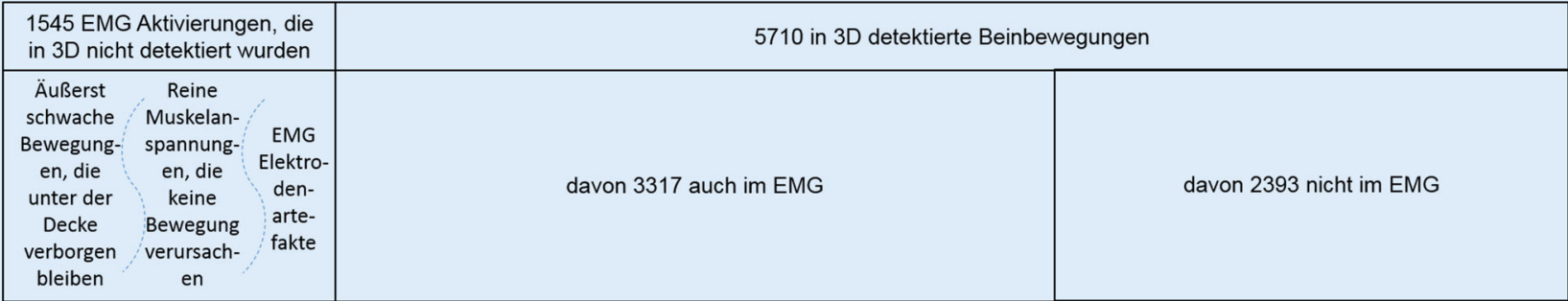

Abb. 3 ॥ Abgrenzung der in 3D und im EMG detektierten und nicht detektierten Bewegungsereignisse

ren. Im Fall (b) lässt sich praktisch nicht entscheiden, ob eine Bewegung stattgefunden hat oder nicht: Im Video bzw. in $3 \mathrm{D}$ ist sie nicht erkennbar, im EMG wiederum könnte es sich um ein Artefakt (Fall a) oder um Fall (c) handeln. Im letzteren Fall (c) wiederum stellt sich die Frage, ob solche rein tonische Anspannungen tatsächlich in den PLM-Index gezählt werden sollen oder nicht. Automatische PSG-Software würde sie mitzählen, 3D nicht.

\section{Diskussion und Schluss- folgerungen}

Untersuchungen über die berührungslose, automatische Detektion von Körperbewegungen im Schlaf wurden bisher nur mit 2D-Infrarotvideos durchgeführt. Mit 3D wurden Atmungsbewegungen des Brustkorbs gemessen. Die vorliegende Arbeit präsentiert erstmals einen Vergleich von Beinbewegungen, die einerseits mit 3D und andererseits mit dem EMG detektiert wurden.

\section{》) Insgesamt ergab die 3D-} Analyse eine realistischere LMAnzahl als die EMG-basierte Zählung

In dieser Studie wurden einzelne Beinbewegungen (LM) betrachtet, da diese die Basis für die darauffolgende Bestimmung periodischer Bewegungsereignisse darstellen. Die Kriterien für die Periodizität können auf Ereignisse aus 3D-Detektionen in gleicher Weise angewandt werden wie auf Ereignisse aus EMG-Detektionen. Hier besteht kein Unterschied zwischen 3D und EMG. Durch 3D-Analyse können aber auch Beinbewegungen detektiert werden, die von anderen Muskeln als den Mm. tibiales anteriores ausgelöst werden, wogegen solche Bewegungen in den entsprechenden EMG-Signalen nicht aufscheinen. Die Untersuchungen haben gezeigt, dass dieser Fall relativ häufig auftritt. Man kann annehmen, dass Bewegungen der anderen Beinmuskeln einen vergleichbaren Krankheitswert repräsentieren wie die der Mm. tibiales anteriores. Bleiben diese Bewegungen unberücksichtigt, wird der PLM-Index deutlich unterschätzt. Insgesamt ergab die 3D-Analyse von Beinbewegungen eine realistischere LM-Anzahl als die EMG-basierte Zählung. Die 3D-Analyse hat daher auch therapeutische Konsequenzen. Die heute geltenden diagnostischen Kriterien (5 bzw. 15 PLM pro Stunde) wären zu überprüfen, wenn auch Beinbewegungen in den PLM-Index einbezogen werden, die von anderen Muskeln als den Mm. tibiales anteriores ausgelöst werden.

Das EMG liefert nur Ja/Nein-Aussagen über das Auftreten einer Bewegung. Art und Ausmaß dieser Bewegung können hingegen nicht zuverlässig abgeleitet werden. Im Vergleich dazu kann man aus der 3D-Analyse wesentlich spezifischere Informationen erhalten: Aus der räumlichen Veränderung der Oberfläche der Bettdecke durch die Bewegung kann man ableiten, wie stark und wohin sich z. B. ein Bein bewegt hat. Dies kann insbesondere bei differenzialdiagnostischen Fragestellungen dienlich sein.

Artefakte von schlechten EMG-Elektrodenkontakten täuschen oft Beinbewegungen vor, die nicht real stattgefunden haben. Dies kann in der Praxis den ge- nannten Effekt der fälschlich nicht detektierten Beinbewegungen in der Summe teilweise kompensieren. Artefakte bringen aber einen starken Unsicherheitsfaktor in die EMG-Auswertungen. Bei der 3D-Analyse können solche Artefakte gar nicht auftreten. 3D bietet daher eine bessere und gleichbleibende Datenqualität.

In der Praxis müssen EMG-Aufzeichnungen heute visuell kontrolliert werden. Mit 3D würde der diesbezügliche Aufwand voraussichtlich sinken: Erstens treten bei 3D keine Artefakte durch schlechte Elektrodenkontakte auf, und zweitens können Ganzkörperbewegungen, die auch Beinbewegungen beinhalten, in 3D automatisch detektiert werden, im EMG aber nicht.

\section{》) Bei der 3D-Analyse können keine Artefakte auftreten}

Ein weiterer Vorteil gegenüber dem EMG ist die berührungslose Messung: Sie vereinfacht die Aufnahmeprozedur, verringert die Sensoren/Kabel am Körper der Patienten und ermöglicht so ungestörten Schlaf. Für Personen, die keine Sensoren am Körper tolerieren, eröffnet die 3DAnalyse eine uneingeschränkte Diagnosemöglichkeit.

Insgesamt könnte die 3D Analyse einige signifikante Vorteile für die Diagnoseunterstützung bei schlafbezogenen Bewegungsstörungen bieten. Die Technologie ist es daher wert, im Hinblick auf eine mögliche Berücksichtigung in diagnostischen Leitlinien näher untersucht und validiert $\mathrm{zu}$ werden. 


\section{Limitationen}

Sowohl das EMG als auch die 3D Messmethode für Beinbewegungen weisen Limitationen auf: Beim EMG sind es die Artefakte und das Ansprechen auf Muskelanspannungen ohne Bewegung. Bei $3 \mathrm{D}$ ist nicht auszuschließen, dass sich ganz schwache Bewegungen unter der Decke nicht auf die Oberfläche der Bettdecke übertragen und somit nicht detektiert werden. Aus den hier dargestellten Ergebnissen ist aber zu erkennen, dass die 3D-basierte Analyse der Realität tatsächlicher Bewegungsereignisse näher kommt als das Tibialis-EMG.

$\mathrm{Da}$ die 3D-basierte Bewegungsdetektion alle Beinbewegungen erfasst, das Tibialis-EMG aber eben nur die von den $\mathrm{Mm}$. tibiales anteriores ausgelösten, liefern die beiden Messmethoden systematisch verschiedene Messwerte und Informationen. Sollte das 3D-Verfahren z. B. zur Bestimmung eines Bewegungsindexes herangezogen werden, müssten neue Bewertungskriterien für die Indexzahl erarbeitet werden.

\section{Fazit für die Praxis}

- 3D-Analyse detektiert auch Beinbewegungen, die von anderen Muskeln als den $\mathrm{Mm}$. tibiales anteriores ausgelöst werden.

- 3D-Analyse reagiert nicht auf subtile tonische Muskelaktivität ohne Bewegung, die aber im EMG sehr wohl die Kriterien der AASM bzw. WASM erfüllen kann.

- Artefakte von schlechten Elektrodenkontakten können bei 3D nicht auftreten.

- Insgesamt ergibt die 3D-Analyse eine realistischere Anzahl von Beinbewegungen als die EMG-basierte Zählung, was auch therapeutische Konsequenzen hat.

- Ein Vorteil gegenüber dem EMG ist die berührungslose Messung: Sie vereinfacht die Prozedur, verringert die Anzahl der Sensoren am Körper und ermöglicht so ungestörten Schlaf.

\section{Korrespondenzadresse

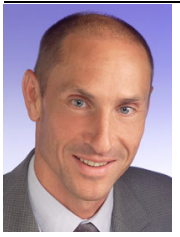 \\ Univ.-Doz. Dipl.-Ing. Dr. H. Garn \\ AIT Austrian Institute of Technology GmbH Donau-City-Straße 1, 1220 Wien, Österreich heinrich.garn@ait.ac.at}

\section{Einhaltung ethischer Richtlinien}

Interessenkonflikt. H. Garn, M. Castrucci, B. Kohn, C. Wiesmeyr, K. Dittrich, G. Kloesch, R. Stepansky, M. Wimmer, A. Stefanic, M. Böck, M. Mandl, O. Ipsiroglu, D. Grossegger, M. Kemethofer und S. Seidel geben an, dass kein Interessenkonflikt besteht.

Die Studie wurde von den zuständigen Ethikkommissionen der Medizinischen Universität Wien ( $\mathrm{Pa}$ tienten der Medizinischen Universität Wien und am Krankenhaus der Barmherzigen Brüder Wien: EK-Nr. 1091/2014) und des Landes Oberösterreich (Patienten des Kepler Universitätsklinikums: EK-Nr. 254) genehmigt. Die Patienten wurden informiert und haben eine Einverständniserklärung unterschrieben.

Open Access. Dieser Artikel wird unter der Creative Commons Namensnennung 4.0 International Lizenz (http://creativecommons.org/licenses/by/4.0/deed. de) veröffentlicht, welche die Nutzung, Vervielfältigung, Bearbeitung, Verbreitung und Wiedergabe in jeglichem Medium und Format erlaubt, sofern Sie den/die ursprünglichen Autor(en) und die Quelle ordnungsgemäßnennen, einen Link zur Creative Commons Lizenz beifügen und angeben, ob Änderungen vorgenommen wurden.

\section{Literatur}

1. Bak JU, Giakoumidis N, Kim G, Dong H, Mavridis N (2012) An intelligent sensing system for sleep motion and stage analysis. Procedia Eng 41:1128-1134

2. Berri RB, Brooks R, Garnaldo CE, Harding SM, Lloyd RM, Marcus CL, Vaughn BV (2015) The AASM manual for the scoring of sleep and associatedevents:rules, terminology and technical specifications, version 2.2. American Association of Sleep Medicine, Darien

3. Cuppens K, Lagae L, Ceulemans B, Van Huffel S, Vanrumste $B$ (2010) Automatic video detection of body movements during sleep based on optical flow in pediatric patients with epilepsy. Med Biol Eng Comput 48:923-931

4. Falie D, David L, Ichim M (2009) Statistical algorithm for detection and screening sleep apnea. IEEE Int'I Symp. Signals, Circuits and Systems, proc, S1-4

5. Falie D, Ichim M (2010) Sleep monitoring and sleep apnea event detection using a 3D camera. IEEE Int'I Conference on Communications, proc, S 177-180

6. Ferri R, Fulda S, Manconi M, Högl B, Ehrmann L, Ferini-Strambi L, Zucconi M (2013) Night-tonight variability of periodic leg movements during sleep in restless legs syndrome and periodic limb movement disorder: Comparison between the periodicity index and the PLMS index. Sleep Med 14:293-296
7. Fong J, Foldvary-Schaefer N (2014) periodic limb movements in sleep (PLMS). Encycl Neurol Sci 3:860-864

8. Frauscher B, Iranzo A, Högl B, Casanova-Molla J, Salamero M, Gschliesser V, Tolosa E, Poewe W, Santamaria J, SINBAR (Sleep Innsbruck Barcelona) group (2008) Quantification of electromyographic activity during REM sleep in multiple muscles in REM sleep behavior disorder. Sleep 31:724-731

9. Garn H, Kohn B, Dittrich K, Wiesmeyr C, Klösch G, Stepansky R, Wimmer M, Ipsiroglu O, Grossegger D, Kemethofer M, Seidel S (2016) 3D detection of periodic limb movements in sleep. 38th Int'I Conference of the IEEE Engineering in Medicine and Biology Society, Orlando, Aug. 2016, S427-430

10. Kohli P, Shotton J (2013) Key developments in human pose estimation for kinect. In: Consumer depth cameras for computer vision. Springer, Berlin, S63-70

11. Liao W-H, Yang C-M (2008) Video-based activity and movement pattern analysis in overnight sleep studies. 19th IEEE Int'I Conf. Pattern Recognition Proc, S1-4

12. Natarajan R (2010) Review of periodic limb movement and restless leg syndrome. J Postgrad Med 56:157-162

13. Payne A, Daniel A, Mehta A, Thompson B, Bamji CS, Snow D, Oshima $H$, Prather L, Fenton M, Kordus $L$, O'Connor P, McCauley R, NayakS, Acharya S, Mehta S, Elkhatib T, Meyer T, O'Dwyer T, Perry T, Chan V-H, Wong V, Mogallapu V, Qian W, Xu Z (2014) A 512×424 CMOS 3D time-of-flight image sensor with multi-frequency photo-demodulation up to $130 \mathrm{MHz}$ and $2 \mathrm{GS} / \mathrm{s}$ ADC. IEEE International SolidState Circuits Conference 2014, S 134-136

14. Sateia MJ (2014) International Classification of Sleep Disorders (ICSD), third edition. Contemp Rev Sleep Med 146(5):1387-1394

15. Scatena M, Dittoni $S$, Maviglia R, Frusciante $R$, Testani E, Vollono C, Losurdo A, Colicchio S, Gnoni V, Labriola C, Farina B, Pennisi MA, Della Marca $G$ (2012) An integrated video-analysis software system designed for movement detection and sleep analysis. Validation of a tool for the behavioural study of sleep. Clin Neurophysiol 123:318-323

16. Takemura Y, Sato J, Nakajima M (2005) A respiratory movement monitoring system using fiber-grating vision sensor for diagnosing sleep apnea. Opt Rev $12: 46-53$

17. Trenkwalder C (2012) Restless-Legs-Syndrom (RLS) und Periodic Limb Movement Disorder (PLMD). Leitlinie der Deutschen Gesellschaft für Neurologie. http://www.awmf.org/ uploads/tx_szleitlinien/030-081I_S1_RestlessLegs-Syndrom_RLS_2012_1_verlaengert.pdf. Zugegriffen:08.05.2017

18. Zucconi M, Ferri R, Allen R, Baier PC, Bruni O, Chokroverty S, Ferini-Strambi L, Fulda S, GarciaBorreguero D, Hening WA, Hirshkowitz M, Högl B, Hornyak M, King M, Montagna P, Parrino L, Plazzi G, Terzano MG (2006) The official World Association of Sleep Medicine (WASM) standards for recording and scoring periodic leg movements in sleep (PLMS) and wakefulness (PLMW) developed in collaboration with a task force from the International Restless Legs Syndrome Study Group (IRLSSG). Sleep Med 7:175-183 